\title{
Electronic and spin structure of the topological insulator $\mathrm{Bi}_{2} \mathrm{Te}_{2.4} \mathrm{Se}_{\mathbf{0 . 6}}$
}

\author{
A. M. Shikin, ${ }^{1}$ I. I. Klimovskikh, ${ }^{1}$ S. V. Eremeev,${ }^{2,3}$ A. A. Rybkina, ${ }^{1}$ M. V. Rusinova, ${ }^{1}$ A. G. Rybkin, ${ }^{1}$ E. V. Zhizhin, ${ }^{1}$ \\ J. Sánchez-Barriga, ${ }^{4}$ A. Varykhalov, ${ }^{4}$ I. P. Rusinov, ${ }^{3}$ E. V. Chulkov, ${ }^{3,5,6}$ K. A. Kokh, ${ }^{3,7,8}$ V. A. Golyashov, ${ }^{9}$ \\ V. Kamyshlov, ${ }^{10}$ and O. E. Tereshchenko ${ }^{3,8,9}$ \\ ${ }^{1}$ Saint Petersburg State University, Saint Petersburg, 198504, Russia \\ ${ }^{2}$ Institute of Strength Physics and Materials Science, Tomsk, 636021 Russia \\ ${ }^{3}$ Tomsk State University, Tomsk, 634050 Russia \\ ${ }^{4}$ Helmholtz-Zentrum Berlin für Materialien und Energie, Elektronenspeicherring BESSY II, Albert-Einstein Str. 15, 12489 Berlin, Germany \\ ${ }^{5}$ Donostia International Physics Center (DIPC), 20018 San Sebastián/Donostia, Basque Country, Spain \\ ${ }^{6}$ Departamento de Física de Materiales UPVIEHU, Centro de Física de Materiales CFM - MPC and Centro Mixto CSIC-UPV/EHU, \\ 20080 San Sebastián/Donostia, Basque Country, Spain \\ ${ }^{7}$ V. S. Sobolev Institute of Geology and Mineralogy, Siberian Branch, Russian Academy of Sciences, Novosibirsk, 630090 Russian Federation \\ ${ }^{8}$ Novosibirsk State University, Novosibirsk, 630090 Russia \\ ${ }^{9}$ A. V. Rzhanov Institute of Semiconductor Physics, Novosibirsk, 630090 Russia \\ ${ }^{10}$ Institute of Automation and Electrometry, Novosibirsk, 630090 Russia
}

(Received 23 November 2013; published 12 March 2014)

\begin{abstract}
High-resolution spin- and angle-resolved photoemission spectroscopy measurements were performed on the three-dimensional topological insulator $\mathrm{Bi}_{2} \mathrm{Te}_{2.4} \mathrm{Se}_{0.6}$, which is characterized by enhanced thermoelectric properties. The Fermi level position is found to be located in the bulk energy gap independent of temperature and it is stable over a long time. Spin textures in the Dirac-cone state at energies above and below the Dirac point as well as in the Rashba-type valence band surface state are observed in agreement with theoretical prediction. The calculations of the surface electronic structure demonstrate that the fractional stoichiometry induced disorder within the Te/Se sublattice does not influence the Dirac-cone state dispersion. In spite of relatively high resistivity, temperature dependence of conductivity shows a weak metallic behavior that could explain the effective thermoelectric properties of the $\mathrm{Bi}_{2} \mathrm{Te}_{2.4} \mathrm{Se}_{0.6}$ compound with the in-plane Seebeck coefficient reaching $-330 \mu \mathrm{V} / \mathrm{K}$ at room temperature.
\end{abstract}

DOI: 10.1103/PhysRevB.89.125416

PACS number(s): 73.20.-r, 79.60.-i

\section{INTRODUCTION}

Rapid development of spintronics aimed at creation of spincurrent devices stimulates intense investigations of systems where nondissipative spin transport, spin-current control, and separation of electrons with different spin orientation are possible. The very promising materials in this respect are topological insulators with unique spin electronic structure. Topological insulators (TIs) are characterized by an insulating energy gap in the bulk and the gapless spin-polarized metallic surface states with linear Dirac-cone-like dispersion in the energy-momentum space with spin of electron locked perpendicular to its momentum (see, for instance, Refs. [1-9]). Owing to the opposite spin orientation for opposite sign of momentum, the topological surface states (TSSs) are protected from backscattering resulting in spin currents with reduced dissipation.

The electronic and spin structures of the surface states for a wide class of TIs are well studied by spin- and angleresolved photoemission spectroscopy (SARPES), in the first instance, for the $\mathrm{Bi}_{2} \mathrm{Se}_{3}$ and $\mathrm{Bi}_{2} \mathrm{Te}_{3}$ compounds [5-7]. These prototypical compounds are characterized by a relatively wide bulk energy gap $\left(\sim 0.3 \mathrm{eV}\right.$ for $\mathrm{Bi}_{2} \mathrm{Se}_{3}$ and $\sim 0.15 \mathrm{eV}$ for $\mathrm{Bi}_{2} \mathrm{Te}_{3}$ ) and a single Dirac cone of the topological surface state at the $\bar{\Gamma}$ point. Typically, they are $n$-type compounds with the Dirac point located inside the bulk energy gap at energies of $0.1-0.4 \mathrm{eV}$ below the Fermi level $[1,5-7,10]$. Such features of the electronic structure are mainly determined by the Se vacancies in $\mathrm{Bi}_{2} \mathrm{Se}_{3}$ and $\mathrm{Bi}-\mathrm{Te}$ antisite defects in
$\mathrm{Bi}_{2} \mathrm{Te}_{3}[7,11]$. Investigations of the Dirac-cone spin structure in these topological insulators confirmed its antisymmetric spin polarization with respect to $k_{\|}[7,12,13]$.

Recently, it was demonstrated that ternary compounds based on binary TIs show a rich variety of TSSs $[3,8,9,14-17]$ including buried TSSs $[3,8]$ and occupied and unoccupied TSSs in local energy gaps $[8,18,19]$. Some of these compounds possess extraordinary wide fundamental energy gaps and nearly ideal Dirac cones [8,15]. It was also shown that the modification of such features of the electronic structure as the position of the Dirac point relative to the Fermi level and to the bulk valence band edge, the absence of bulk conduction band states at the Fermi level, etc., can be efficiently controlled by means of a variation of the third component concentration $[1,5-7,9,20]$ or by diluted metal atom doping [7].

The ternary topological compounds $\mathrm{Bi}_{2} \mathrm{Se}_{2} \mathrm{Te}$ and $\mathrm{Bi}_{2} \mathrm{Te}_{2} \mathrm{Se}$ were studied by SARPES [20], ARPES with circular light polarization [21], and angle-resolved two-photon photoemission [18] with analysis of their spin texture. In contrast to the binary compounds, $\mathrm{Bi}_{2} \mathrm{Te}_{2} \mathrm{Se}$ is characterized by a higher insulating gap resistivity [11,22]. In such compounds, the central $\mathrm{Te}(\mathrm{Se})$ layer in $\mathrm{Bi}_{2} \mathrm{Te}_{3}\left(\mathrm{Bi}_{2} \mathrm{Se}_{3}\right)$ is substituted for the $\mathrm{Se}$ (Te) layer. It was shown [9] that the change of the concentration of Bi and Se in ternary Bi-Te-Se TIs leads to the shift of both the Fermi level position into the bulk energy gap and the position of the Dirac point relative to the valence band, which result in the change of the surface-state contribution to the conductivity. Ternary Bi-Te-Se TIs exhibit a temperature-dependent 
resistivity typical for bulk insulators [22]. The low-temperature resistivity for these compounds is much as two to three orders of magnitude higher than that in $\mathrm{Bi}_{2} \mathrm{Se}_{3}$ [22], meaning that they are characterized by much better insulating properties as compared to prototypical TIs. Moreover, as it was shown in Refs. [23,24], the $\mathrm{Bi}_{2} \mathrm{Te}_{2.4} \mathrm{Se}_{0.6}$ alloy has the widest band gap among $\mathrm{Bi}_{2} \mathrm{Te}_{3-x} \mathrm{Se}_{x}$ and exhibits enhanced thermoelectric properties and good quantum transport properties [11,25-27], thus, the detailed analysis of the electronic structure of this compound is required.

Aside from the topological surface state in the bulk band gap, Rashba-type spin-split surface states reside in the valence and conduction bands of $\mathrm{Bi}_{2} \mathrm{Se}_{3}, \mathrm{Bi}_{2} \mathrm{Te}_{3}$, and $\mathrm{Sb}_{2} \mathrm{Te}_{3}[5,18,28]$. In addition to these states, quantized Rashba-type states below the conduction band bottom (inside the bulk band gap) and M-shaped states inside the valence band local gap can arise as a result of adsorption of residual gases and of a variety other species. Such states can be formed due to confinement of the bulk conduction and valence band states in a quantum well which develops in a thin surface layer if band bending produced by adsorption [29-31] or intercalation of adsorbates into the van der Waals spacings [32,33] are significant.

This work aims to provide a detailed experimental and theoretical investigation of the electronic and spin structures of topological and Rashba-type surface states in the $\mathrm{Bi}_{2} \mathrm{Te}_{2.4} \mathrm{Se}_{0.6}$ topological insulator. We show that this compound has remarkable electronic properties, namely, the ideal spin-polarized Dirac cone lies at the Fermi level where neither bulk conduction band states nor quantized Rashba split states are present. Our calculation results based on density functional theory (DFT) nicely reproduce the ARPES spectra and demonstrate that the slope of the Dirac state dispersion is determined by the Se concentration without any effect of disorder within the Te/Se sublattice. SARPES and transport measurements allow us to suggest the use of this ternary compound as a system where a spin current can be generated from controlled thermal or electrical gradients.

\section{EXPERIMENTAL AND COMPUTATIONAL DETAILS}

The experiments were carried out at the beamlines UE112-SGM and U125/2-SGM of Helmholtz-Zentrum Berlin (BESSY II) with linearly and circularly polarized light using a Scienta R4000 energy analyzer and a Mott spin detector operated at $26 \mathrm{keV}$. The light incidence angle with respect to the sample normal was $45^{\circ}$. SARPES measurements were performed with linear $p$-polarized undulator radiation with the polarization plane oriented parallel to the spin of the studied states. Thereat, the variation of the polar angle (corresponding to the variation of $k_{\|}$) took place in the plane perpendicular to the plane of the incident light polarization and parallel to the slit of the analyzer. The schematic drawing of the geometry of the experiment is shown in Fig. 1. For the measurements with circular light polarization (clockwise and anticlockwise), the same geometry of the experiment was used. Part of these experiments were carried out at the Research Resource Center of Saint Petersburg State University "Physical methods of surface investigation" using the same detection geometry in combination with a monochromatized discharge He-lamp providing 21.2-eV photons. In these experiments, a slightly



FIG. 1. (Color online) Schematic drawing of the geometry of the experiment. Variation of $k_{\|}$takes place by tilting the sample in the plane perpendicular to the plane of polarization of the incident light. Linear $p$ and circularly polarized photons are used. The measured in-plane orientation of spin is parallel to the plane of the incident light polarization.

different light incidence angle of $65^{\circ}$ relative to the surface normal was used.

The $n$-doped samples with stoichiometry of $\mathrm{Bi}_{2} \mathrm{Te}_{2.4} \mathrm{Se}_{0.6}$ were grown from a previously synthesized mixture of $\mathrm{Bi}_{2} \mathrm{Te}_{3}$ and $\mathrm{Bi}_{2} \mathrm{Se}_{3}$ compounds using a modified vertical Bridgman method [34]. Before measurements, the samples were cleaved in situ at room temperature in ultrahigh vacuum. For measurements at cryogenic temperature $(18 \mathrm{~K})$ the samples were cleaved at $18 \mathrm{~K}$ directly at the manipulator. The base pressure in the experimental chamber during the experiments was below $1-2 \times 10^{-10}$ mbar.

The Hall resistance $R_{y x}$ and the resistance $R_{x x}$ were measured in the Hall bar geometry by a standard six-probe method on rectangular samples on which the contacts were made with indium at the perimeter. The magnetic field was swept between $2 \mathrm{~T}$ at fixed temperatures and was always applied perpendicular to the samples. The Seebeck coefficient was measured by connecting one side of the sample to a heated metal block and the other side to a heat sink kept at room temperature, maintaining a temperature difference between both sides below $5 \mathrm{~K}$.

The electronic band calculations on ordered $\mathrm{Bi}_{2} \mathrm{Te}_{3}$ and $\mathrm{Bi}_{2} \mathrm{Te}_{2} \mathrm{Se}$ [Figs. 2(a) and 2(b)] were performed within the DFT formalism as implemented in VASP [35,36]. We use the all-electron projector augmented wave (PAW) $[37,38]$ basis sets with the generalized gradient approximation (GGA) of Perdew, Burke, and Ernzerhof (PBE) [39] to model the exchange-correlation (XC) potential. Relativistic effects, including spin-orbit interaction (SOI), were fully taken into account.

The atomic positions were obtained during a relaxation procedure at a fixed volume until forces became less than $1.0 \times 10^{-6} \mathrm{eV} / \AA$. Our total energy estimations within a supercell approach revealed that substitution of a Te atom 


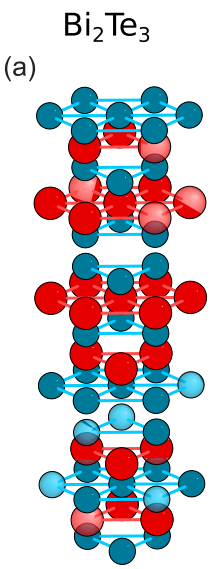

(b)

$\mathrm{Bi}_{2} \mathrm{Te}_{2} \mathrm{Se}$

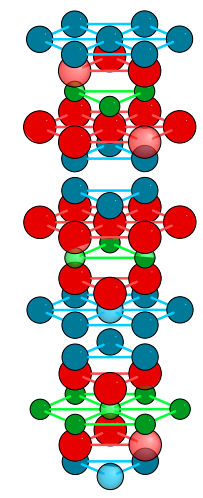

$\mathrm{Bi} \bigcirc \mathrm{Te} \bigcirc \mathrm{Se}$ (c)

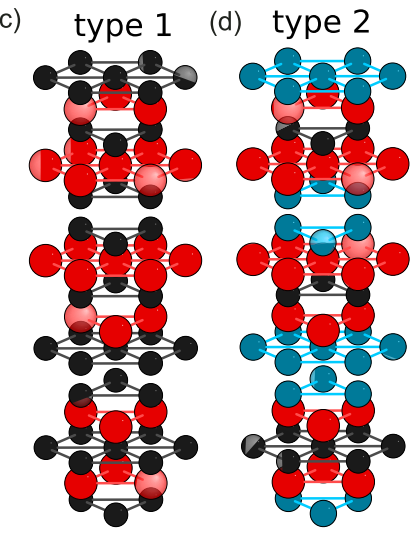

type 1: $0.8 \mathrm{Te} / 0.2 \mathrm{Se}$

type 2: $0.4 \mathrm{Te} / 0.6 \mathrm{Se}$

FIG. 2. (Color online) Atomic structure of $\mathrm{Bi}_{2} \mathrm{Te}_{3}$ (a), $\mathrm{Bi}_{2} \mathrm{Te}_{2} \mathrm{Se}$ (b), and $\mathrm{Bi}_{2} \mathrm{Te}_{2.4} \mathrm{Se}_{0.6}$ with different distribution of Se atoms in the Te/Se sublattice: (c) homogeneous distribution; (d) distribution of Se atoms in the central layer of QL.

in the central atomic layer of a quintuple layer $(\mathrm{QL})$ of $\mathrm{Bi}_{2} \mathrm{Te}_{3}$ by a $\mathrm{Se}$ atom has the energy gain of $\sim 40 \mathrm{meV}$ with respect to substitution in the outermost atomic layer. To treat the disordered $\mathrm{Bi}_{2} \mathrm{Te}_{2.4} \mathrm{Se}_{0.6}$, we employed a virtual crystal approximation (VCA) as implemented in the ABINIT code [40], where the configuration averaged potential of a "gray" atom occupying a site in the $\mathrm{Te} / \mathrm{Se}$ sublattice is defined as a mixture $V_{\mathrm{VCA}}=x V_{\mathrm{Te}}+(1-x) V_{\mathrm{Se}}$ of $\mathrm{Te}\left(V_{\mathrm{Te}}\right)$ and $\mathrm{Se}$ $\left(V_{\mathrm{Se}}\right)$ pseudopotentials. To address the effect of Se disordering within the Te/Se sublattice on the bulk and surface electronic structure of $\mathrm{Bi}_{2} \mathrm{Te}_{2.4} \mathrm{Se}_{0.6}$, two extreme types of disorder were examined [see Figs. 2(c) and 2(d)]: (1) a case where Se atoms are homogeneously distributed over three Te layers within the QL $(x=0.8)$ and (2) a case where Se atoms substitute Te atoms in the central atomic layer of the QL only $(x=0.4)$, being the outermost atomic layers composed of Te atoms. We used GGA-PBE Hartwigsen-Goedecker-Hutter (HGH) [41] relativistic norm-conserving pseudopotentials which include the SOI. In the VCA calculations, the atomic positions were optimized too.

To simulate the (0001) surface, we consider six quintuple layer slabs. A vacuum spacer of $\sim 12 \AA$ was included to ensure a negligible interaction between neighboring slabs.

\section{ELECTRONIC AND SPIN STRUCTURE}

Figures 3(a) and 3(b) show the ARPES spectra for $\mathrm{Bi}_{2} \mathrm{Te}_{2.4} \mathrm{Se}_{0.6}$ measured along the $\bar{\Gamma}-\overline{\mathrm{K}}$ direction of the surface Brillouin zone (SBZ). Measurements were carried out directly after sample cleavage at $18 \mathrm{~K}$ [Fig. 3(a)] and at room temperature [Fig. 3(b)] using $p$-polarized light and a photon energy of $52 \mathrm{eV}$. One can observe the topological surface state with the Dirac point located at a binding energy of about $0.28-0.33 \mathrm{eV}$ which is comparable to the one obtained for $\mathrm{Bi}_{2} \mathrm{Se}_{2} \mathrm{Te}$ and $\mathrm{Bi}_{2} \mathrm{Te}_{2} \mathrm{Se}$ compounds [20]. Similarly, in Ref. [21], the position of the Dirac point for $\mathrm{Bi}_{2} \mathrm{Te}_{2} \mathrm{Se}$ was estimated at a binding energy of about $0.23 \mathrm{eV}$. The linear

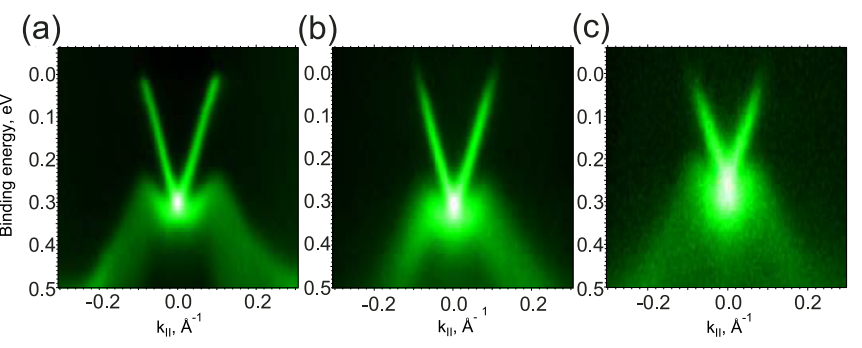

FIG. 3. (Color online) ARPES spectra along the $\overline{\mathrm{K}}-\bar{\Gamma}-\overline{\mathrm{K}}$ line acquired at $52-\mathrm{eV}$ photon energy for freshly cleaved surface at (a) cryogenic $(18 \mathrm{~K})$ and (b) room temperatures, and (c) for the aged surface.

dispersion of the topological surface state is well visible in Fig. 3 both at $18 \mathrm{~K}$ and at room temperature. Variation of the photon energy leads to a modulation of the photoemission intensity for both the topological surface state and the valence band states [Figs. 4(a) and 4(b)]. Measurements using the discharge He lamp at 21.2-eV photon energy showed almost the same position of the Dirac point ( $\sim 0.28 \mathrm{eV})$ [see Fig. 4(c)].

A shift of the Dirac point toward higher binding energies and the appearance of the occupied states near the bottom of the bulk conduction band are typically observed for BiTe-Se topological compounds even at cryogenic temperatures [29-31]. In these works, the observed states appeared around the bottom of the conduction band and below the upper edge of the surface-projected valence band gap. These states appear at the surfaces of topological compounds due to the band-bending effect caused by the $n$-type doping provided by adsorption of residual gases or due to intercalation of impurities into the van der Waals spacings. In contrast, in $\mathrm{Bi}_{2} \mathrm{Te}_{2.4} \mathrm{Se}_{0.6}$ we occasionally observed the shift of the Dirac point toward lower binding energies within $50 \mathrm{meV}$ [Fig. 3(c)]. For the perfectly cleaved samples, the surface Fermi level position was constant for a long time reflecting the surface inertness to adsorbates [42]. We did not observe any states at the Fermi level related to the conduction band in the spectra using a wide range of photon energies including $21.2 \mathrm{eV}$. The surface stability can be explained by the low concentration of surface defects in our samples grown by modified Bridgman method $[42,43]$. The energy shift of the Dirac point toward the Fermi level with time can be related to the adsorption of the residual oxygen-contained gases at the surface, in contrast to the case of $\mathrm{CO}$ adsorption which leads to a shift of the Dirac point in the opposite direction [31]. Owing to the surface
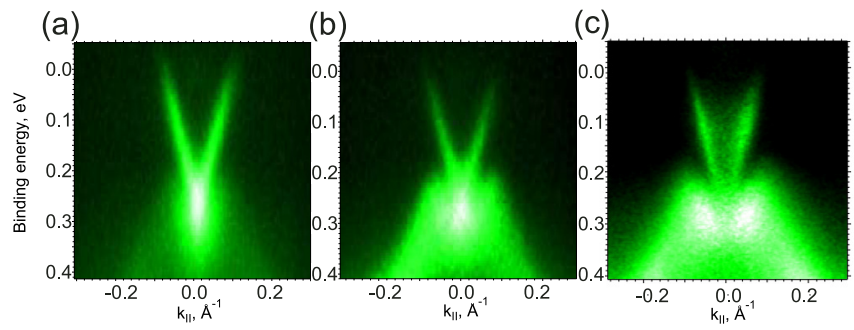

FIG. 4. (Color online) ARPES spectra measured at room temperature with different photon energies: (a) $57 \mathrm{eV}$ and (b) $47 \mathrm{eV}$ using synchrotron radiation and (c) at $21.2 \mathrm{eV}$ using the discharge He lamp. 
- $\mathrm{Bi}_{2} \mathrm{Te}_{2} \mathrm{Se}$ - $\mathrm{Bi}_{2} \mathrm{Te}_{24} \mathrm{Se}_{06}$ (type 1)

(a) $-\mathrm{Bi}_{2} \mathrm{Te}_{3}-\mathrm{Bi}_{2} \mathrm{Te}_{2.4} \mathrm{Se}_{0.6}$ (type 2)

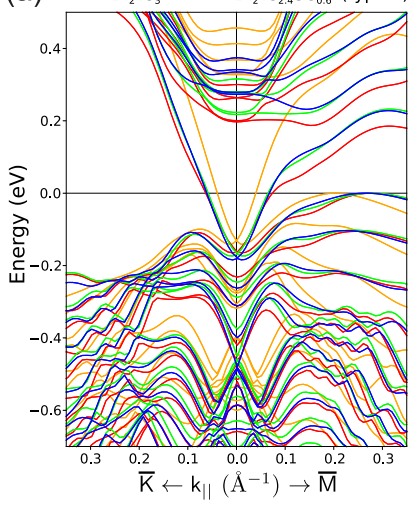

(b)

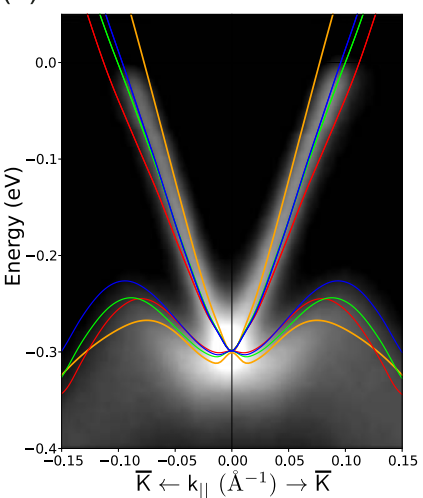

(a)

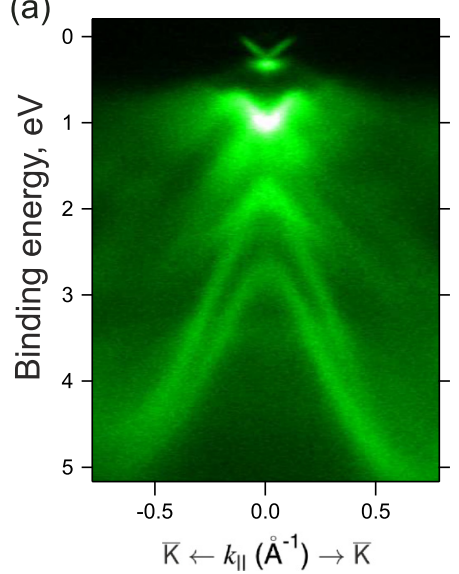

(b)

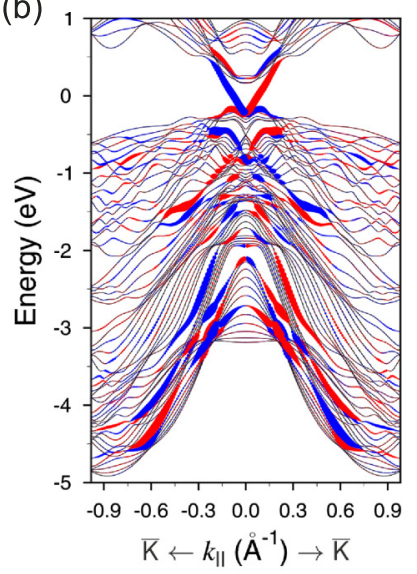

FIG. 5. (Color online) (a) Band spectra of $6 \mathrm{QL}$ slabs of $\mathrm{Bi}_{2} \mathrm{Te}_{3}$, $\mathrm{Bi}_{2} \mathrm{Te}_{2} \mathrm{Se}$, and $\mathrm{Bi}_{2} \mathrm{Te}_{2.4} \mathrm{Se}_{0.6}$ with different Se disordering in the latter system; zero energy corresponds to the top of the bulk valence band. (b) ARPES measurement at 52-eV photon energy on $\mathrm{Bi}_{2} \mathrm{Te}_{2.4} \mathrm{Se}_{0.6}$ with overlaid surface band structure obtained from DFT calculations.

inertness, the Fermi level lies always (even after long-time exposure to residual gas in ultrahigh vacuum conditions) inside the bulk energy gap.

Next, we discuss the calculated energy spectra of $\mathrm{Bi}_{2} \mathrm{Te}_{2.4} \mathrm{Se}_{0.6}$ by comparing it with the ordered $\mathrm{Bi}_{2} \mathrm{Te}_{3}$ and $\mathrm{Bi}_{2} \mathrm{Te}_{2} \mathrm{Se}$ compunds. In Fig. 5(a), one can see that the top of the bulk valence band states of $\mathrm{Bi}_{2} \mathrm{Te}_{2.4} \mathrm{Se}_{0.6}$ is located in the $\bar{\Gamma}-\bar{M}$ plane at $k_{\|} \approx 0.27 \AA^{-1}$ as it is the case in $\mathrm{Bi}_{2} \mathrm{Te}_{3}$. The Dirac point position in $\mathrm{Bi}_{2} \mathrm{Te}_{2.4} \mathrm{Se}_{0.6}$ slightly varies with the type of Se disordering ( -167 and $-179 \mathrm{meV}$ for types 1 and 2, respectively) and lies deeper relative to the upper edge of the valence band states as compared to the ordered compounds. The figure demonstrates also the variation of the $\bar{\Gamma}$ edge of the bulk conduction band on the type of Se disordering. The lowest unoccupied $\bar{\Gamma}$ state for type-1 case lies just $\sim 20 \mathrm{meV}$ above the $\bar{\Gamma}$ edge in $\mathrm{Bi}_{2} \mathrm{Te}_{3}$, while it is $56 \mathrm{meV}$ higher for type-2 $\mathrm{Bi}_{2} \mathrm{Te}_{2.4} \mathrm{Se}_{0.6}$. The calculated Dirac-cone dispersions along the $\overline{\mathrm{K}}-\bar{\Gamma}-\overline{\mathrm{K}}$ direction for $\mathrm{Bi}_{2} \mathrm{Te}_{3}, \mathrm{Bi}_{2} \mathrm{Te}_{2} \mathrm{Se}$, and $\mathrm{Bi}_{2} \mathrm{Te}_{2.4} \mathrm{Se}_{0.6}$, superimposed on the experimental ARPES results, are shown in Fig. 5(b). Here, the binding energies of the calculated Dirac points are aligned with respect to the experimental one. As one can see, in $\mathrm{Bi}_{2} \mathrm{Te}_{2.4} \mathrm{Se}_{0.6}$ the group velocity of the surface states above the Dirac point has approximately intermediate value between those in $\mathrm{Bi}_{2} \mathrm{Te}_{3}$ and $\mathrm{Bi}_{2} \mathrm{Te}_{2} \mathrm{Se}$. The dispersion of the Dirac cone is almost independent of the type of Se disordering demonstrating an excellent agreement with the experiment. The ARPES results in Fig. 6(a) reveal several bright spectral features dispersing deep into the valence band which can be mostly identified with the calculated spin-polarized trivial surface states shown in Fig. 6(b).

In addition to the direct measurement of the spin structure by means of SARPES, we have used angle-resolved photoemission with left- and right-circular polarization of the incident light. These types of measurements are much less time consuming in comparison to the direct method of spin-resolved photoemission. The possibility of using circularly polarized photons to obtain information about the peculiar spin structure of TSSs was investigated in several papers [21,44-49] for both the prototypical TIs $\mathrm{Bi}_{2} \mathrm{Se}_{3}$ and $\mathrm{Bi}_{2} \mathrm{Te}_{3}$ [44-49] and the mixed

FIG. 6. (Color online) ARPES (a) and DFT (b) spectra of the occupied electronic states of $\mathrm{Bi}_{2} \mathrm{Te}_{2.4} \mathrm{Se}_{0.6}$ along $\overline{\mathrm{K}}-\bar{\Gamma}-\overline{\mathrm{K}}$; in panel (b) circles represent weights of the states, localized in the outermost QL, multiplied by value of in-plane spin components (red and blue colors denote positive and negative values of spin, respectively).

compound $\mathrm{Bi}_{2} \mathrm{Te}_{2} \mathrm{Se}$ [21]. Part of these works have shown that the ARPES signal obtained from circular dichroism in the angular distribution (CDAD) can not be interpreted only in terms of the initial states, as it depends on the coupling between initial and final states via photoemission matrix elements [21,49], on the interaction between bulk and surface states [21], and on the photon energy [21,49]. In particular, the effect of CDAD in ARPES has been shown to be very sensitive to the symmetry of the final states reached at different photon energies [49], a fact which leads to multiple reversals of the circular dichroism asymmetry with photon energy when $d$-like final states are reached in the photoemission process [49]. On the other hand, circularly polarized photons might be used for inducing a spin current with a well-separated spin structure, as it was noted in Ref. [50]. In this context, photoelectron spin manipulation with light polarization has been recently observed in SARPES experiments on the topological surface state of $\mathrm{Bi}_{2} \mathrm{Se}_{3}$ using linearly and circularly polarized $6-\mathrm{eV}$ laser photons $[45,46]$. It is important to mention that under the condition of $d$-like final states, the recently observed spin manipulation with light polarization contributes unobservably to the measured photoelectron spin polarization. [46] Thus, in order to perform SARPES experiments, measurements of the circular dichroism asymmetry were used as a preliminary test to identify the photon energies where the final states entering the photoemission matrix elements are predominantly $d$ like [49].

Using the reversal of the CDAD effect with photon energy as a probe of the character of the final states, we have identified that this condition is fulfilled at $52-\mathrm{eV}$ photon energy where the circular dichroism asymmetry near the Fermi level reaches a maximum absolute value of up to $\sim 40 \%$. The corresponding photoemission spectra measured at this photon energy along the $\overline{\mathrm{K}}-\bar{\Gamma}-\overline{\mathrm{K}}$ direction at $18 \mathrm{~K}$ using clockwise (right-handed) and anticlockwise (left-handed) circular light polarization are presented in Figs. 7(a) and 7(b), respectively. Figure 7(c) shows the corresponding momentum distribution curves (MDCs) extracted at different binding energies for 



FIG. 7. (Color online) Circular dichroism of the topological surface states of $\mathrm{Bi}_{2} \mathrm{Te}_{2.4} \mathrm{Se}_{0.6}$ measured along the $\overline{\mathrm{K}}-\bar{\Gamma}-\overline{\mathrm{K}}$ direction of the SBZ. Angle-resolved photoemission intensity $\left(E-k_{\|}\right)$measured for (a) clockwise (right) and (b) counterclockwise (left) circular polarization of the incident light. (c) MDCs extracted at different binding energies for opposite circular polarizations and (d) the corresponding dichroism dispersion.

both clockwise (blue color) and anticlockwise (red color) circular polarizations. One can see in Fig. 7(c) that in the upper Dirac-cone region $\left(E=0.1-0.2 \mathrm{eV} ; k_{\|}= \pm 0.1 \AA^{-1}\right.$ ), the MDCs exhibit a pronounced intensity asymmetry upon reversal of the light helicity. Simultaneously, such asymmetry can also be distinguished for the states which are located near the lower edge of the local gap in the surface-projected valence band energy gap $\left(E=0.7-0.8 \mathrm{eV} ; k_{\|}=0.3-0.5 \AA^{-1}\right)$, while the exact intensity asymmetry for the lower Dirac cone is not resolved in present circular dichroism measurements probably due to the overlap with bulk valence band states [see the corresponding dichroism dispersion in Fig. 7(d)].

The resulting spin structure for the Dirac-cone topological surface and the valence band states measured by means of SARPES is shown in Fig. 8. Similar to the results shown in Fig. 7, a photon energy of $52 \mathrm{eV}$ was used. The measured spin orientation ( $S_{\text {in-plane }}$ ) was locked perpendicular to $k_{\|}$ (Fig. 1). The $p$ polarization of the light was oriented parallel to the spin directions to additionally minimize the influence of the geometry of the experiment on the relation between the resulting spin-polarized photoemission spectra and the initialstate spin structure [44-46]. Figure 8(a) shows that the spin polarization in the upper Dirac cone is inverted relative to the surface normal. For the lower Dirac cone, the sign of the spin polarization is opposite to that of the upper Dirac cone. Thus, the presented spin structure of the topological surface state is typical for $\mathrm{Bi}_{2} \mathrm{Te}_{3}-(\mathrm{Bi}-\mathrm{Te}-\mathrm{Se})-\mathrm{Bi}_{2} \mathrm{Se}_{3}$ series. Figure 8(b) shows the spin-resolved energy dispersion curves (EDCs) for the Rashba-type valence band surface states predicted by the DFT calculation [Fig. 6(b)]. These states exhibit noticeable spin polarization which increases with increasing $k_{\|}$. The sign of the spin polarization for the inner Rashba-type branch is the same as for the upper Dirac cone that correlates well with the theoretical calculations of the initial-state spin structure. Note
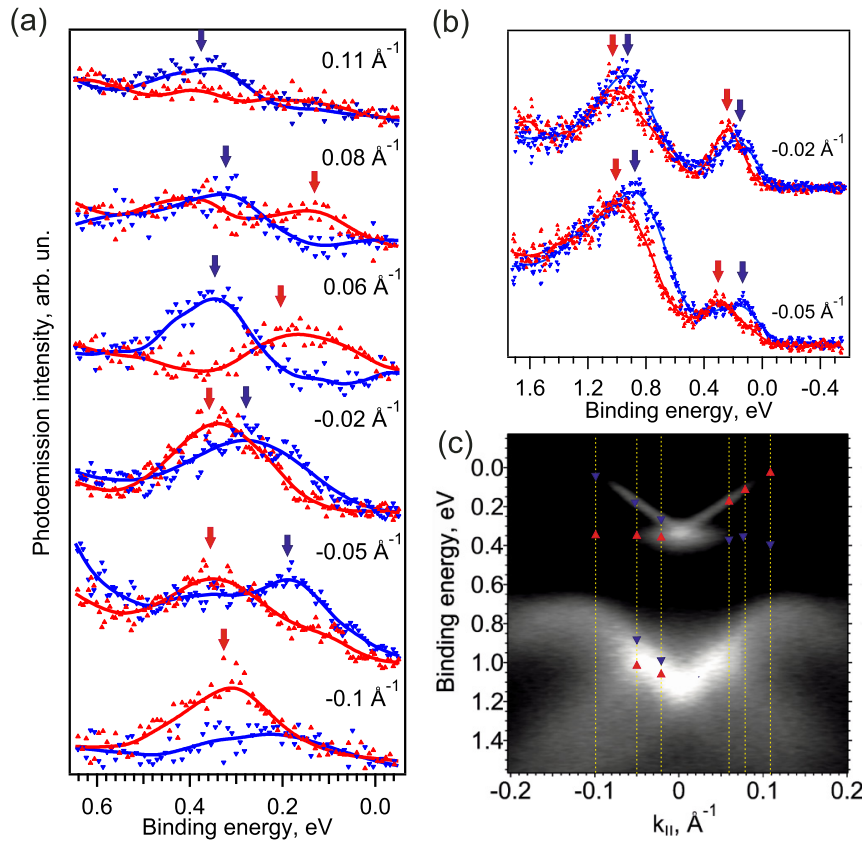

FIG. 8. (Color online) Spin-resolved EDCs measured at 52-eV photon energy at room temperature in the $\overline{\mathrm{K}}-\bar{\Gamma}-\overline{\mathrm{K}}$ direction of the SBZ (a) the Dirac-cone topological surface state and (b) the region including the Rashba-type surface state located near the lower edge of the surface-projected valence band gap. Panel (c) summarizes the spin structure of the Dirac and Rashba-type states.

that the measurements of the circular dichroism asymmetry for these states show a completely different behavior and therefore this method is also not suitable to correctly identify the spin structure of the Rashba-type valence band surface states.

\section{TRANSPORT MEASUREMENTS}

The measured in-plane Seebeck coefficient of the $\mathrm{Bi}_{2} \mathrm{Te}_{2.4} \mathrm{Se}_{0.6}(0001)$ sample demonstrated a rather high value, reaching approximately $-330 \mu \mathrm{V} / \mathrm{K}$ at room temperature, suggesting an $n$-type conductivity. This value exceeded the in-plane Seebeck coefficient of $\mathrm{Bi}_{2} \mathrm{Te}_{3}$, equal to $-280 \mu \mathrm{V} / \mathrm{K}$.

Figures 9(a) and 9(b) show the temperature dependence of the magnetoresistance $\mathrm{MR}(\Delta R / R)$ and the Hall resistance. These data highlight several regimes. In the temperature range between 100 and $300 \mathrm{~K}$, both the MR and the Hall resistance are quadratic and linear with the applied magnetic field up to $2 \mathrm{~T}$, respectively, and follow Boltzmann transport theory. Between 100 and $25 \mathrm{~K}$, the Hall resistance continues to increase and shows nonlinear behavior for applied magnetic fields higher than $1 \mathrm{~T}$, reflecting the appearance of a second carrier channel with higher mobility. The deviation of the MR dependence from a quadratic function at $25 \mathrm{~K}$ confirms this suggestion. Below $25 \mathrm{~K}$, both the Hall resistance and the MR decrease, a fact that can be explained by the compensation effect induced by holes. The observed maximum of MR is $10 \%$ at $25 \mathrm{~K}$. Figure 9 (c) shows the changes of the electrical conductivity and Hall concentration as a function of temperature. The magnitude of the resistivity at room temperature is equal to $100 \mathrm{~m} \Omega \mathrm{cm}$, which is comparable with that found in more complex $\mathrm{Bi}_{2-x} \mathrm{Sb}_{x} \mathrm{Te}_{3-y} \mathrm{Se}_{y}$ series 
(a)

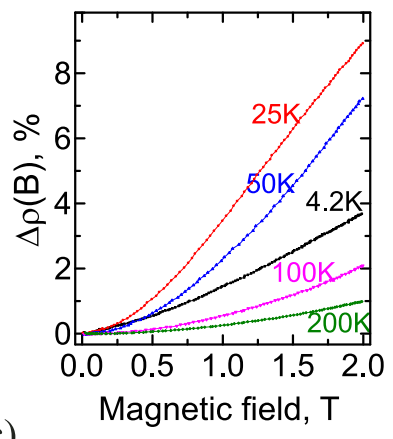

(c)

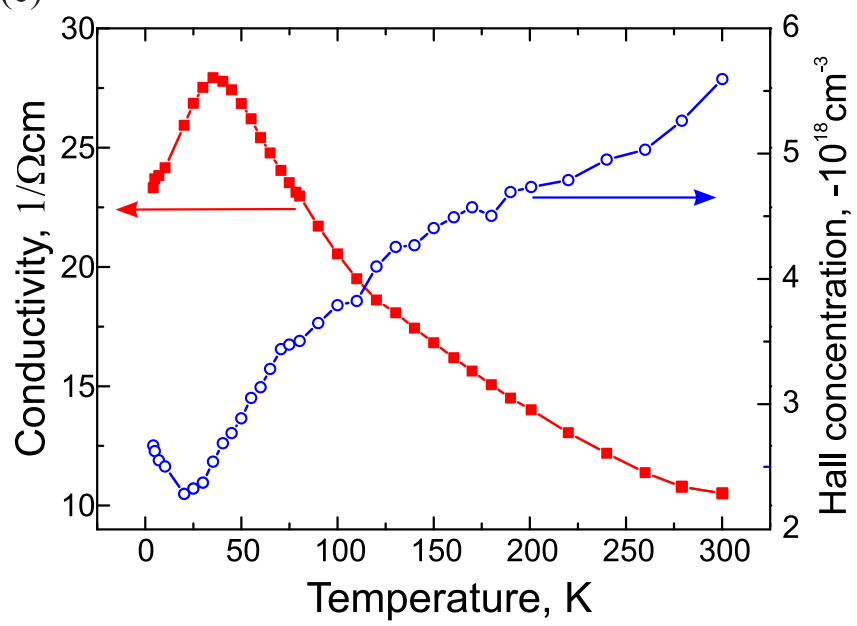

FIG. 9. (Color online) Temperature dependence of the magnetoresistance $\operatorname{MR}(\Delta R / R)$ (a), Hall resistance (b), and electrical conductivity and Hall concentration (c).

of compositions [11]. Nevertheless, it can be seen that as the temperature decreases, the conductivity increases leading to metallic behavior, while the Hall concentration decreases leading to semiconductorlike behavior. This discrepancy can be explained by the increase of the electrons mobility with decreasing temperature. At a temperature below $25 \mathrm{~K}$, the conductivity decreases while the concentration behaves in the opposite way. The conductivity shows the $\ln T$ decrease between 4 and $25 \mathrm{~K}$, which can be due to the two-dimensional electron-electron interaction effect observed in prototypical TIs near the weakly disordered regime [51]. The changes of the transport properties below $25 \mathrm{~K}$ can be explained by assuming the bulk Fermi level shift within the bulk energy gap with decreasing temperature.

The fact that the Fermi level in $\mathrm{Bi}_{2} \mathrm{Te}_{2.4} \mathrm{Se}_{0.6}$ crosses only the TSSs makes this compound a promising candidate for the observation of nondissipative surface spin transport. In this respect, applying an electrical or thermal gradient, for instance in the $k_{x}$ direction, should lead to the formation of electrically (thermally) driven spin currents which propagate through the topological surface state in a direction which is parallel to the in-plane spin orientation, which at the same time must be locked perpendicular to the momentum of electrons moving under the applied electric (thermal) gradient.

Typically, the spin selection in spintronic devices based on the spin filtering effect is produced with assistance of ferromagnetic metals (FM). These metals are characterized by a significantly different density of states at the Fermi level for states with opposite spin orientation. Injection of electrons with selected spin orientation from these states could create the required spin selection in the forming spin current. However, TIs can create the spin current itself without using FM. In TIs, the spin selection takes place due to the unique spin structure of the nondegenerated Dirac-cone surface states which have the spin orientation locked to the momentum of the in-plane moving electrons, a fact which allows us to suggest a generator of the spin current without using FM. Thereat, not only an electrical or thermal gradient, but also excitation by circularly polarized laser light (as in Ref. [50]) can be used for creation of such a spin current. In this case, for the registration and transfer of the spin current formed at the surface, one can propose to use nonmagnetic Pt contacts. $\mathrm{Pt}$ is characterized by an enhanced density of states at the Fermi level for opposite spin orientations, which is one of the necessary preconditions for effective spin injection, and by a high value of the Hall conductivity $[52,53]$. In particular for $\mathrm{Bi}_{2} \mathrm{Te}_{2.4} \mathrm{Se}_{0.6}$, our experiments reveal that contributions of the bulk conduction band states at the Fermi level are absent and, therefore, the formed spin current is expected to be fully determined by the unique properties of the TSSs.

\section{SUMMARY}

In conclusion, a systematic investigation of the electronic and spin structure of the topological insulator $\mathrm{Bi}_{2} \mathrm{Te}_{2.4} \mathrm{Se}_{0.6}$ with enhanced thermoelectric properties has been presented. Using spin- and angle-resolved photoemission, it was shown that the $\mathrm{Bi}_{2} \mathrm{Te}_{2.4} \mathrm{Se}_{0.6}$ compound is characterized by the ideallike spin-polarized Dirac-cone topological surface state. The Fermi level position was found to be located inside the bulk energy gap, stable over a long time and independent of the sample temperature. Our DFT calculation results are in good agreement with the ARPES spectra, demonstrating that the Dirac state dispersion is determined by the Se concentration and not affected by disorder within the Te/Se sublattice. Aside from the topological surface state in the bulk gap, Rashba-type spin-polarized surface states were observed near the lower edge of the surface-projected valence band energy gap. Transport experiments revealed that in spite of relative high value of resistivity, the temperature dependence of the conductivity in this compound follows a weak metallic behavior, a fact that implies its effective thermoelectric properties.

\section{ACKNOWLEDGMENTS}

This work was supported by grants of Saint Petersburg State University for scientific investigations and Russian Foundation for Basic Research (RFBR) projects (Projects No. 12-0200226, No. 13-02-91327, and No. 13-02-12110 ofi-m) and performed in framework of collaboration between the Deutsche Forschungsgemeinschaft and Russian Foundation for Basic Research (Projects No. 13-02-91336 and No. RA 1041/3-1). Authors acknowledge Helmholtz-Zentrum Berlin for financial and technical support. M.V.R and I.I.K acknowledge for support the German-Russian Interdisciplinary Science Center (G-RISC) funded by the German Federal Foreign Office via the German Academic Exchange Service (DAAD) 
[1] M. Z. Hasan and C. L. Kane, Rev. Mod. Phys. 82, 3045 (2010).

[2] X.-L. Qi and S.-C. Zhang, Physics Today 63(1), 33 (2010).

[3] T. Okuda and A. Kimura, J. Phys. Soc. Jpn. 82, 021002 (2013).

[4] Y. Ando, J. Phys. Soc. Jpn. 82, 102001 (2013).

[5] Y. Xia, D. Qian, D. Hsieh, L. Wray, A. Pal, H. Lin, A. Bansil, D. Grauer, Y. S. Hor, R. J. Cava, and M. Z. Hasan, Nat. Phys. 5, 398 (2009).

[6] Y. L. Chen, J. G. Analytis, J.-H. Chu, Z. K. Liu, S.-K. Mo, X. L. Qi, H. J. Zhang, D. H. Lu, X. Dai, Z. Fang, S. C. Zhang, I. R. Fisher, Z. Hussain, and Z.-X. Shen, Science 325, 178 (2009).

[7] D. Hsieh, Y. Xia, D. Qian, L. Wray, J. H. Dil, F. Meier, J. Osterwalder, L. Pattney, J. G. Checkelsky, N. P. Ong, A. V. Fedorov, H. Lin, A. Bansil, D. Grauer, Y. S. Hor, R. J. Cava, and M. Z. Hasan, Nature (London) 460, 1101 (2009).

[8] S. V. Eremeev, G. Landolt, T. V. Menshchikova, B. Slomski, Y. M. Koroteev, Z. S. Aliev, M. B. Babanly, J. Henk, A. Ernst, L. Patthey, A. Eich, A. A. Khajetoorians, J. Hagemeister, O. Pietzsch, J. Wiebe, R. Wiesendanger, P. M. Echenique, S. S. Tsirkin, I. R. Amiraslanov, J. H. Dil, and E. V. Chulkov, Nat. Commun. 3, 635 (2012).

[9] M. Neupane, S.-Y. Xu, L. A. Wray, A. Petersen, R. Shankar, N. Alidoust, C. Liu, A. Fedorov, H. Ji, J. M. Allred, Y. S. Hor, T.-R. Chang, H.-T. Jeng, H. Lin, A. Bansil, R. J. Cava, and M. Z. Hasan, Phys. Rev. B 85, 235406 (2012).

[10] K. Kuroda, M. Arita, K. Miyamoto, M. Ye, J. Jiang, A. Kimura, E. E. Krasovskii, E. V. Chulkov, H. Iwasawa, T. Okuda, K. Shimada, Y. Ueda, H. Namatame, and M. Taniguchi, Phys. Rev. Lett. 105, 076802 (2010).

[11] Z. Ren, A. A. Taskin, S. Sasaki, K. Segawa, and Y. Ando, Phys. Rev. B 82, 241306(R) (2010).

[12] C. Jozwiak, Y. L. Chen, A. V. Fedorov, J. G. Analytis, C. R. Rotundu, A. K. Schmid, J. D. Denlinger, Y.-D. Chuang, D.-H. Lee, I. R. Fisher, R. J. Birgeneau, Z.-X. Shen, Z. Hussain, and A. Lanzara, Phys. Rev. B 84, 165113 (2011).

[13] Z.-H. Pan, E. Vescovo, A. V. Fedorov, D. Gardner, Y. S. Lee, S. Chu, G. D. Gu, and T. Valla, Phys. Rev. Lett. 106, 257004 (2011)

[14] T. V. Menshchikova, S. V. Eremeev, Yu. M. Koroteev, V. M. Kuznetsov, and E. V. Chulkov, JETP Lett. 93, 15 (2011).

[15] T. V. Menshchikova, S. V. Eremeev, and E. V. Chulkov, JETP Lett. 94, 106 (2011).

[16] S. Muff, F. von Rohr, G. Landolt, B. Slomski, A. Schilling, R. J. Cava, J. Osterwalder, and J. H. Dil, Phys. Rev. B 88, 035407 (2013).

[17] T. Okuda, T. Maegawa, M. Ye, K. Shirai, T. Warashina, K. Miyamoto, K. Kuroda, M. Arita, Z. S. Aliev, I. R. Amiraslanov, M. B. Babanly, E. V. Chulkov, S. V. Eremeev, A. Kimura, H. Namatame, and M. Taniguchi, Phys. Rev. Lett. 111, 206803 (2013).

[18] D. Niesner, Th. Fauster, S. V. Eremeev, T. V. Menshchikova, Yu. M. Koroteev, A. P. Protogenov, E. V. Chulkov, O. E. Tereshchenko, K. A. Kokh, O. Alekperov, A. Nadjafov, and N. Mamedov, Phys. Rev. B 86, 205403 (2012).

[19] S. V. Eremeev, I. V. Silkin, T. V. Menshchikova, A. P. Protogenov, and E. V. Chulkov, JETP Lett. 96, 780 (2013).

[20] K. Miyamoto, A. Kimura, T. Okuda, H. Miyahara, K. Kuroda, H. Namatame, M. Taniguchi, S. V. Eremeev, T. V. Menshchikova, E. V. Chulkov, K. A. Kokh, and O. E. Tereshchenko, Phys. Rev. Lett. 109, 166802 (2012).
[21] M. Neupane, S. Basak, N. Alidoust, S.-Y. Xu, Chang Liu, I. Belopolski, G. Bian, J. Xiong, H. Ji, S. Jia, S.-K. Mo, M. Bissen, M. Severson, H. Lin, N. P. Ong, T. Durakiewicz, R. J. Cava, A. Bansil, and M. Z. Hasan, Phys. Rev. B 88, 165129 (2013).

[22] D. O. Scanlon, P. D. C. King, R. P. Singh, A. de la Torre, S. McKeown Walker, G. Balakrishnan, F. Baumberger, and C. R. A. Catlow, Adv. Mater. 24, 2154 (2012).

[23] J. G. Austin and A. Sheard, J. Electr. Control 3, 236 (1957).

[24] H. J. Goldsmid, in Materials used in Semiconductor Devices, edited by C. A. Hogarth (Wiley, London, 1965), p. 165.

[25] Huiwen Ji, J. M. Allred, M. K. Fuccillo, M. E. Charles, M. Neupane, L. A. Wray, M. Z. Hasan, and R. J. Cava, Phys. Rev. B 85, 201103(R) (2012).

[26] K.-W. Cho and I.-H. Kim, Mater. Lett. 59, 966 (2005).

[27] A. Kadhim, A. Hmood, Y. Abu Hassan, Solid State Commun. 166, 44 (2013).

[28] C. Pauly, G. Bihlmayer, M. Liebmann, M. Grob, A. Georgi, D. Subramaniam, M. R. Scholz, J. Sánchez-Barriga, A. Varykhalov, S. Blügel, O. Rader, and M. Morgenstern, Phys. Rev. B 86, 235106 (2012).

[29] Z.-H. Zhu, G. Levy, B. Ludbrook, C. N. Veenstra, J. A. Rosen, R. Comin, D. Wong, P. Dosanjh, A. Ubaldini, P. Syers, N. P. Butch, J. Paglione, I. S. Elfimov, and A. Damascelli, Phys. Rev. Lett. 107, 186405 (2011).

[30] M. S. Bahramy, P. D. C. King, A. de la Torre, J. Chang, M. Shi, L. Patthey, G. Balakrishnan, Ph. Hofmann, R. Arita, N. Nagaosa, and F. Baumberger, Nat. Commun. 3, 1159 (2012).

[31] P. D. C. King, R. C. Hatch, M. Bianchi, R. Ovsyannikov, C. Lupulescu, G. Landolt, B. Slomski, J. H. Dil, D. Guan, J. L. Mi, E. D. L. Rienks, J. Fink, A. Lindblad, S. Svensson, S. Bao, G. Balakrishnan, B. B. Iversen, J. Osterwalder, W. Eberhardt, F. Baumberger, and Ph. Hofmann, Phys. Rev. Lett. 107, 096802 (2011).

[32] S. V. Eremeev, M. G. Vergniory, T. V. Menshchikova, A. A. Shaposhnikov, and E. V. Chulkov, New J. Phys. 14, 113030 (2012).

[33] Chaoyu Chen, Shaolong He, Hongming Weng, Wentao Zhang, Lin Zhao, Haiyun Liu, Xiaowen Jia, Daixiang Mou, Shanyu Liu, Junfeng He, Yingying Peng, Ya Feng, Zhuojin Xie, Guodong Liu, Xiaoli Dong, Jun Zhang, Xiaoyang Wang, Qinjun Peng, Zhimin Wang, Shenjin Zhang, Feng Yang, Chuangtian Chen, Zuyan Xu, Xi Dai, Zhong Fang, and X. J. Zhou, Proc. Natl. Acad. Sci. USA 109, 3694 (2012).

[34] K. A. Kokh, B. G. Nenashev, A. E. Kokh, and G. Yu. Shvendenkov, J. Cryst. Growth 275, e2129 (2005).

[35] G. Kresse and J. Hafner, Phys. Rev. B 48, 13115 (1993).

[36] G. Kresse and J. Furthmüller, Comput. Mater. Sci. 6, 15 (1996).

[37] P. E. Blöchl, Phys. Rev. B 50, 17953 (1994).

[38] G. Kresse and D. Joubert, Phys. Rev. B 59, 1758 (1999).

[39] J. P. Perdew, K. Burke, and M. Ernzerhof, Phys. Rev. Lett. 77, 3865 (1996).

[40] X. Gonze, B. Amadon, P.-M. Anglade, J.-M. Beuken, F. Bottin, P. Boulanger, F. Bruneval, D. Caliste, R. Caracas, M. Cote, T. Deutsch, L. Genovese, Ph. Ghosez, M. Giantomassi, S. Goedecker, D. R. Hamann, P. Hermet, F. Jollet, G. Jomard, S. Leroux, M. Mancini, S. Mazevet, M. J. T. Oliveira, G. Onida, Y. Pouillon, T. Rangel, G.-M. Rignanese, D. Sangalli, R. Shaltaf, M. Torrent, M. J. Verstraete, G. Zerah, and J. W. Zwanziger, Comput. Phys. Commun. 180, 2582 (2009); http://www.abinit.org. 
[41] C. Hartwigsen, S. Goedecker, and J. Hutter, Phys. Rev. B 58, 3641 (1998).

[42] V. A. Golyashov, K. A. Kokh, S. V. Makarenko, K. N. Romanyuk, I. P. Prosvirin, A. V. Kalinkin, O. E. Tereshchenko, A. S. Kozhukhov, S. V. Eremeev, S. D. Borisova, and E. V. Chulkov, J. Appl. Phys. 112, 113702 (2012).

[43] V. V. Atuchin, V. A. Golyashov, K. A. Kokh, I. V. Korolkov, A. S. Kozhukhov, V. N. Kruchinin, S. V. Makarenko, L. D. Pokrovsky, I. P. Prosvirin, K. N. Romanyuk, and O. E. Tereshchenko, Cryst. Growth Des. 11, 5507 (2011).

[44] C. H. Park and S. G. Louie, Phys. Rev. Lett. 109, 097601 (2012).

[45] C. Jozwiak, C.-H. Park, K. Gotlieb, C. Hwang, D.-H. Lee, S. G. Louie, J. D. Denlinger, C. R. Rotundu, R. J. Birgeneau, Z. Hussain, and A. Lanzara, Nat. Phys. 9, 293 (2013).

[46] J. Sánchez-Barriga, A. Varykhalov, J. Braun, S.-Y. Xu, N. Alidoust, O. Kornilov, J. Minár, K. Hummer, G. Springholz, G. Bauer, R. Schumann, L. V. Yashina, H. Ebert, M. Z. Hasan, and O. Rader, arXiv:1310.1160 [Phys. Rev. X (to be published)].
[47] Y. Ishida, H. Kanto, A. Kikkawa, Y. Taguchi, Y. Ito, Y. Ota, K. Okazaki, W. Malaeb, M. Mulazzi, M. Okawa, S. Watanabe, C.-T. Chen, M. Kim, C. Bell, Y. Kozuka, H. Y. Hwang, Y. Tokura, and S. Shin, Phys. Rev. Lett. 107, 077601 (2011).

[48] Y. H. Wang, D. Hsieh, D. Pilon, L. Fu, D. R. Gardner, Y. S. Lee, and N. Gedik, Phys. Rev. Lett. 107, 207602 (2011).

[49] M. R. Scholz, J. Sánchez-Barriga, J. Braun, D. Marchenko, A. Varykhalov, M. Lindroos, Yung Jui Wang, Hsin Lin, A. Bansil, J. Minár, H. Ebert, A. Volykhov, L. V. Yashina, and O. Rader, Phys. Rev. Lett. 110, 216801 (2013).

[50] K. Ando, M. Morikawa, T. Trypiniotis, Y. Fujikawa, C. H. W. Barnes, and E. Saitoh, Appl. Phys. Lett. 96, 082502 (2010).

[51] Shao-Pin Chiu and Juhn-Jong Lin, Phys. Rev. B 87, 035122 (2013).

[52] G. Y. Guo, S. Murakami, T.-W. Chen, and N. Nagaosa, Phys. Rev. Lett. 100, 096401 (2008).

[53] H. Kontani, M. Naito, D. S. Hirashima, K. Yamada, and J. Inoue, J. Phys. Soc. Jpn. 76, 103702 (2007). 